\title{
Philosophiques
}

\section{Le programme philosophique sous-jacent aux Remarques philosophiques}

\section{João Vergílio Gallerani Cuter et Bento Prado Neto}

Volume 39, numéro 1, printemps 2012

La période intermédiaire de Wittgenstein

URI : https://id.erudit.org/iderudit/1011610ar

DOI : https://doi.org/10.7202/1011610ar

Aller au sommaire du numéro

Éditeur(s)

Société de philosophie du Québec

ISSN

0316-2923 (imprimé)

1492-1391 (numérique)

Découvrir la revue

Citer cet article

Gallerani Cuter, J. V. \& Prado Neto, B. (2012). Le programme philosophique sous-jacent aux Remarques philosophiques. Philosophiques, 39(1), 57-74.

https://doi.org/10.7202/1011610ar
Résumé de l'article

Les Remarques philosophiques sont la première tentative de mettre en oeuvre le programme qui découle de l'échec du projet du Tractatus Logico-Philosophicus. Le noyau de ce programme est donné par l'abandon de l'analyse des nombres avancée dans le Tractatus. Wittgenstein se rend compte que les nombres doivent se trouver à la base même du langage, dans la structure des propositions élémentaires. En même temps, il se rend compte qu'il est impossible de fournir la logique sousjacente au langage avant d'avoir procédé à l'analyse. Le projet d'une investigation logique des phénomènes s'imposait, donc, même après l'abandon de l'idée d'un langage phénoménologique. 


\title{
Le programme philosophique sous-jacent aux Remarques philosophiques'
}

\author{
JOÃO VERGÍLIO GALLERANI CUTER \\ Université de São Paulo, Brésil \\ jv-cuter@uol.com.br
}

\begin{abstract}
RÉSUMÉ. - Les Remarques philosophiques sont la première tentative de mettre en oeuvre le programme qui découle de l'échec du projet du Tractatus LogicoPhilosophicus. Le noyau de ce programme est donné par l'abandon de l'analyse des nombres avancée dans le Tractatus. Wittgenstein se rend compte que les nombres doivent se trouver à la base même du langage, dans la structure des propositions élémentaires. En même temps, il se rend compte qu'il est impossible de fournir la logique sousjacente au langage avant d'avoir procédé à l'analyse. Le projet d'une investigation logique des phénomènes s'imposait, donc, même après l'abandon de l'idée d'un langage phénoménologique.
\end{abstract}

\begin{abstract}
The Philosophical Remarks is a first attempt at pursuing the philosophical agenda determined by the failure of the Tractarian project. The central point of this agenda is the abandonment of the analysis of number that had been given in the Tractatus. Wittgenstein realizes that numbers should be brought back to the basis of language, as part of the structure of the elementary propositions themselves. At the same time, he realizes that it is impossible to give the logic underlying our language before the process of logical analysis is carried out. He undertakes the project of a logical investigation of phenomena even after giving up the idea of a phenomenological language.
\end{abstract}

Dans les semaines qui ont précédé notre colloque sur «le Wittgenstein intermédiaire», j'ai invité les participants à suggérer quelques questions que nous pourrions discuter ensemble dans les conférences. Le professeur Sören Stenlund m'a envoyé une lettre proposant un problème qui, me semble-t-il, a une importance centrale pour l'interprétation des Philosophische Bemerkungen. Voici le passage qui nous intéresse:

J'ai toujours eu l'impression que les remarques que l'on trouve dans les Philosophische Bemerkungen, notamment celles que nous discuterons, sont pour la plupart très difficiles, et plus obscures et impénétrables que les remarques que l'on trouve dans ses autres textes publiés (comme la Grammaire philosophique et les Remarques sur les fondements des mathématiques).

Nombre de fois, cette difficulté s'est présentée à moi comme une difficulté de comprendre quels sont les problèmes qu'il se pose. Je me suis souvent demandé: par rapport à quel horizon de problèmes telle ou telle remarque devient-elle intéressante et significative? Quand et où tel ou tel problème nous pose-t-il une véritable difficulté, un puzzle?

1. Je remercie tous les participants du II Middle Wittgenstein Colloquium pour leurs observations lors de la discussion du texte qui a été à l'origine de cet article. 
J'ai parfois l'impression que tout se passe comme s'il avait une espèce de programme caché avec un horizon qu'il tient pour acquis. Et c'est peut-être là que plusieurs de ces problèmes surgissent. Mais quel est ce horizon? Considérez, par exemple, les remarques au sujet de la grammaire de l'espace visuel. J'ai l'impression que ce sujet appartient à un contexte plus large et qu'il fait partie, d'une façon ou d'une autre, d'un projet plus ample. Ou est-ce vraiment un sujet philosophique censé être intéressant de son propre chef, autant, par exemple, que ses remarques plus tardives sur la couleur?

Je ne prétends pas avoir de réponse précise sur ces questions difficiles. Dans un certain sens, elles présupposent une interprétation cohérente et soutenue du livre entier, et il y a une bonne partie de ce texte dont j'avoue que le sens m’échappe. Néanmoins, j’ai une hypothèse générale au sujet du programme philosophique sous-jacent aux Philosophische Bemerkungen, et je pense qu'il sera utile de l'articuler aussi clairement que possible afin d'en extraire un point de départ, voire un guide.

Si j'ai raison, le programme sous-jacent à cette œuvre a clairement une question centrale autour de laquelle s'organisent toutes les autres questions qui y sont posées ainsi que les essais de solution qui y sont ébauchés. Les Bemerkungen furent écrites dans le contexte d'une crise dans le projet philosophique du Tractatus. Selon Wittgenstein lui-même, son premier livre a dû être abandonné (ou au moins profondément reformulé) parce que ses vues sur le nombre étaient insoutenables. C'est du moins le diagnostic qu'il fait dans "Some Remarks on Logical Form», et je pense que nous devrions le prendre au sérieux. Je ne suggère pas par là que tout et chaque élément de ce petit texte "faible et peu caractéristique ${ }^{2}$ " ait été incorporé aux Bemerkungen ou aux autres textes de la même période. Ce n'est certainement pas vrai. Nous pouvons, par exemple, identifier dans cette conférence non prononcée les échos du projet de "construire un langage phénoménologique» qui sera abandonné peu après. D’ailleurs, dans cette conférence, Wittgenstein est beaucoup plus à l'aise avec le concept de "proposition élémentaire » qu'il ne le sera dans les années suivantes. Mais il ne reviendra jamais sur la critique de ses anciennes vues sur les nombres. Examinons le passage crucial et comparons-le à d'autres passages où exactement les mêmes idées réapparaissent - presque in extenso parfois.

Après l'énoncé d'une thèse d'allure très tractatuséenne selon laquelle «si [...] nous essayons d'en venir à une analyse effective, nous découvrons des formes logiques qui n'ont que très peu de ressemblance avec les normes

2. Selon Elizabeth Anscombe, tels sont les adjectifs que Wittgenstein a employés pour décrire son article (cf. Essays on Wittgenstein's Tractatus, Irving M. Copi, and Robert W. Beard, eds., London, Routledge and Kegan Paul, 1966, p. 31). Du point de vue de sa philosophie tardive, les mêmes adjectifs peuvent également être employés pour décrire tout ce qu'il a écrit pendant la période intermédiaire, avec cette seule différence que la conférence avait déjà été publiée quand il parlait à Anscombe, contrairement aux autres textes. 
du langage ordinaire ${ }^{3}$ ", et après avoir donné à titre d'exemples «les formes de l'espace et du temps avec toute la diversité des objets spatiaux et temporels, comme les couleurs, les sons, etc. ", Wittgenstein fait (plutôt solennellement) sa "première remarque bien précise sur l'analyse logique des phénomènes réels". Pour la représentation de ces phénomènes, dit-il, les «nombres (rationnels et irrationnels) entrent dans la structure des propositions atomiques ellesmêmes ». Le ton solennel est ici tout à fait à sa place. Il serait difficile de penser à une rupture plus radicale par rapport au Tractatus. Ce qui est en jeu, ce n'est pas un petit détail insignifiant de son premier livre, mais le cœur même de tout le projet. Dans le Tractatus, les nombres n'étaient que des dispositifs d'abréviation, dont nous pouvons parfaitement nous passer sans que le langage perde sa capacité d'expression. À l'époque, Wittgenstein croyait que tout — absolument tout ce que nous pouvons dire en utilisant des nombres - pourrait parfaitement être dit sans qu'on les emploie: les quantificateurs imbriqués pourraient s'acquitter de la besogne, comme nous le verrons à l'instant. Mais avant d'y venir, il est sans doute utile de suivre les explications que Wittgenstein lui-même nous donne au sujet des raisons qui l'ont amené à un geste si radical ${ }^{4}$ :

Imaginez un système de coordonnées cartésiennes, quelque chose comme des fils croisés tracés dans notre champ visuel, et une échelle fixée arbitrairement. Il est clair que nous pouvons alors décrire la forme et la position de toute tache de couleur se trouvant dans notre champ visuel en énonçant des nombres dont la signification est relative au système de coordonnées et à l'unité choisie. De plus, il est clair que cette description possédera la multiplicité logique correcte, et qu'une description qui aurait une multiplicité moindre ne suffira pas. Un exemple simple consisterait à représenter une tache $\mathrm{P}$ par l'expression « $[6-9,3-8]$ » et à représenter une proposition concernant cette tache, $\mathrm{P}$ est rouge par exemple, par le symbole « $[6-9,3-8] \mathrm{R}$ », où « $R$ » est un terme non encore analysé (« 6-9 » et «3-8» représentant respectivement l'intervalle continu entre ces nombres).

Une illustration ajoutée au texte ${ }^{5}$ nous aide à comprendre le genre de situation qui serait décrite par une telle proposition (il faut simplement imaginer la tache comme étant rouge au lieu de noire):

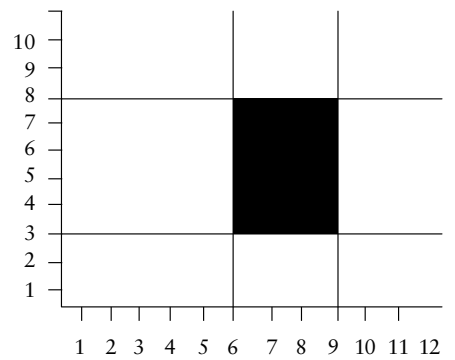

3. Wittgenstein, I993, p. 3 I ; trad. fr., p. 20-22. L'article a d'abord été publié dans les Proceedings of the Aristotelian Society, Supp. vol. 9, 1929, et aussi plusieurs autres fois (par exemple, dans le volume organisé par Copi et Beard que nous avons cité plus haut).

4. Ibid., p. 3 I (c'est moi qui souligne); trad. fr. p. 22.

5. Ibid., p. 3 I ; trad. fr. p. 24. 
Mais tout d'abord, il y a une question préliminaire à résoudre: ce genre d'analyse est-il compatible avec le Tractatus? Est-ce que «[ $\left.6 \begin{array}{lll}6 & 9.3 & 8\end{array}\right]$ $\mathrm{R}$ » pourrait être une proposition élémentaire? Pourrait-elle être prise (d'un point de vue tractatuséen) comme résultat final de l'analyse logique du langage? Non, évidemment, et cela pour plus d'une raison. En premier lieu, il s'agit là d'attributions de couleurs, et selon le Tractatus celles-ci ne peuvent pas être élémentaires ${ }^{6}$. De plus, il s'agit de nombres, et ceux-ci ne peuvent pas être utilisés comme matériau de base pour la construction de propositions élémentaires - ce ne sont pas des noms ${ }^{7}$. Comme nous le verrons à l'instant, pour le Tractatus, l'analyse fait disparaître les nombres. Ils peuvent faire partie de propositions complexes, mais ils y sont introduits comme dispositifs d'abréviation indiquant l'utilisation implicite de quantificateurs imbriqués. Ainsi, la nouveauté ici n'est pas la présence des nombres: c'est plutôt l'idée que des propositions avec des nombres pourraient être élémentaires, d'où il suit par une conséquence immédiate et nécessaire que (au moins dans certains contextes) l'analyse ne fait pas disparaître les nombres. Wittgenstein est tout à fait explicite à ce sujet ${ }^{8}$ :

L'intervention des nombres dans les formes des propositions atomiques n'est pas seulement, à ce qu'il me semble, un trait caractéristique qui appartiendrait à un symbolisme particulier, mais un trait essentiel et en conséquence inévitable de la représentation. Et les nombres doivent intervenir dans ces formes lorsque - ainsi que nous le dirions dans la langue ordinaire - nous traitons de propriétés qui admettent une gradation, par exemple de propriétés comme la longueur d'un intervalle, la hauteur d'un son, la luminosité ou la rougeur d'une nuance de couleur, etc.

Or un trait important des rapports de degré est qu'ils sont incompatibles les uns avec les autres. "Une nuance de couleur ne peut pas avoir simultanément deux degrés différents de luminosité ou de rougeur, un son ne peut pas avoir deux intensités différentes, etc.» Voilà ce que le Tractatus aurait accordé de bonne grâce: "[Q]ue [...] deux couleurs soient ensemble en un même lieu du champ visuel est impossible [...], car c'est la structure logique de la couleur qui l'exclut»(6.375 I). C'est exactement pour cette raison que des attributions de couleur ne peuvent être considérées comme des propositions élémentaires. Étant complexes, elles devraient être analysées, et leurs propriétés logiques devraient être présentées en termes d'applications successives d'opérations de vérité en partant du niveau élémentaire.

6. Tractatus, 6.375 I : «Il est clair que le produit logique de deux propositions élémentaires ne peut être ni une tautologie ni une contradiction. Énoncer qu'un point dans le champ visuel a dans le même temps deux couleurs différentes est une contradiction. "

7. Ibid., 4.22: "Der Elementarsatz besteht aus Namen». Pour un compte rendu du concept tractatuséen du nombre, voir Frascolla, I994, chapitre I; Marion, I998, chapitre 2; Potter, 2000, chapitre 6.

8. Wittgenstein, I985, p. 32 (c'est moi qui souligne); trad. fr. p. 24-26. 
Comment effectuer une telle analyse? Nous ne trouverons aucune réponse dans le Tractatus, mais nous pouvons y trouver une piste. Après avoir admis qu'il est contradictoire de dire d'une tache dans mon champ visuel qu'elle est rouge et verte en même temps, il dit $^{9}$ :

Réfléchissons à la manière dont cette contradiction se présente en physique; à peu près ainsi: une particule ne peut pas avoir au même instant deux vitesses; c'est-à-dire qu'elle ne peut pas être au même instant en deux lieux; c'est-à-dire que des particules, en des lieux différents en un seul moment de temps, ne peuvent être identiques.

Les détails qui concernent l'opposition entre particules et ondes sont sans importance ici. Une théorie physique sur le monde physique n'est employée dans ce passage que pour établir une analogie: Wittgenstein est en train de nous dire qu'il est aussi contradictoire d'énoncer l'existence d'une tache dans mon champ visuel qui serait simultanément rouge et verte, qu'il est contradictoire (logiquement contradictoire) de déclarer qu'un objet physique a deux couleurs en même temps. Si nous nous demandons comment cette contradiction se présente dans une théorie physique, nous verrons qu'elle implique des attributions incompatibles de nombres: deux vitesses différentes, deux coordonnées spatiales différentes pour le même objet. Dans tous ces contextes, il s'agit de "propriétés qui admettent une gradation", c'est-à-dire des "propriétés comme la longueur d'un intervalle, la hauteur d'un son, la luminosité ou la rougeur d'une nuance de couleur, etc.» (pour citer encore le même passage de la conférence de I929). Dans tous ces cas, il s'agit d'attributions de nombres incompatibles entre elles, exactement comme quand nous attribuons différentes vitesses ou positions dans l'espace et dans le temps à une seule et même chose. Ainsi, dans le Tractatus, Wittgenstein pensait également à analyser les énoncés sur les couleurs en termes d'attributions de nombres, même si à cette époque il ne semblait avoir aucune idée arrêtée sur la façon précise d'effectuer cette analyse. L'espace et le temps avaient exactement ce même horizon d'analyse. À un certain niveau de l'analyse, nous aurions quelque chose de très semblable à «[6-9.3-8] R». La différence, comme nous l'avons dit, est que le récit tractatuséen ne pourrait pas s'arrêter à ce moment-là. Il devrait pouvoir nous dire comment ramener des attributions de nombres (peu importe les formes spécifiques qu'elles peuvent prendre) à des fonctions de vérité de propositions élémentaires. Dans la période intermédiaire, avec «[6-9.3-8] R» l'histoire n'est certes pas finie, mais seulement pour ce qui est de ce nom de couleur « R », qui attend encore son analyse. Comme nous venons de le voir, tant dans le Tractatus que dans les Bemerkungen, les couleurs devraient être analysées en termes de nombres. Ainsi, «[6-9.3-8] $\mathrm{R}$ » deviendrait quelque chose comme «[6-9.3-8] $(\mathrm{a}, \mathrm{b}, \mathrm{c})$ », où « $\mathrm{a}$ ", « $\mathrm{b}$ » et «c » sont des nombres 
associés à un certain système de coordonnées chromatique (le système octaédrique, par exemple, comme nous le verrons à l'instant). Encore une fois, dans les Bemerkungen, l'histoire finirait là, alors que dans le Tractatus il faut que la trame se déploie jusqu'à ce qu'on arrive aux propositions élémentaires cachées sous ces nombres et ces coordonnées. Mais là il nous dit que les nombres doivent être présents dès le début, en tant que parties composantes de la structure des propositions élémentaires elles-mêmes. Pourquoi cette exigence?

Selon Wittgenstein, c'est parce que les nombres tractatuséens étaient assez bons pour compter, mais complètement incapables de mesurer. Le passage suivant de la conférence sur la forme logique est bien connu ${ }^{10}$ :

L'on pourrait penser - et je pensais moi-même ainsi naguère - qu'un énoncé exprimant le degré d'une qualité serait analysable en un produit logique d'énoncés quantitatifs simples et en un énoncé supplémentaire les complétant. De la même façon, je pourrais décrire ce que ma poche contient en disant: «Elle contient un penny, un shilling, deux clefs et rien d'autre.» «Rien d'autre » est l'énoncé supplémentaire qui complète la description. Mais cela ne marchera pas, s'agissant d'analyser un énoncé portant sur le degré. En effet, si nous appelons l'unité, l'unité de luminosité par exemple, $b$, et $\mathrm{E}$ (b) l'énoncé qui pose que l'entité $\mathrm{E}$ possède cette luminosité, alors la proposition $\mathrm{E}(\mathbf{2} \mathrm{b})$, qui dit que E possède deux degrés de luminosité, devrait être analysable en produit logique $\mathrm{E}(\mathrm{b}) \& \mathrm{E}(\mathrm{b})$, mais celui-ci est égal à $\mathrm{E}(\mathrm{b})$; si, d'un autre côté, nous tentons de distinguer les unités les unes des autres et écrivons en conséquence $\mathrm{E}(2 \mathrm{~b})=\mathrm{E}\left(\mathrm{b}^{\prime}\right) \& \mathrm{E}\left(\mathrm{b}^{\prime}\right.$ "), nous admettons deux unités différentes de luminosité; et alors, lorsqu'une entité possède une unité, la question pourrait se poser de savoir laquelle des deux — b' ou $b$ " - elle possède; ce qui est manifestement absurde.

Il ne s'agit pas là d'une subtilité tirée d'un texte quelque peu déviant de cette période. En consultant le début du huitième chapitre des Bemerkungen, on trouve exactement la même idée ${ }^{11}$ :

[D]ifférents degrés de rouge sont incompatibles les uns avec les autres. On pourrait imaginer une explication en disant par exemple que l'addition la plus minime d'une quantité de rouge entraîne un certain degré de rouge. Mais qu'est-ce que cela signifie de dire qu'il y a par exemple cinq degrés de telles quantités de rouge? Qu'il y ait la quantité $\mathrm{n}^{\circ} \mathrm{I}$, puis la quantité $\mathrm{n}^{\circ}$ 2, etc., jusqu'à la quantité ${ }^{\circ} 5$, cela ne peut évidemment pas être un produit logique; sinon, comment ces quantités se différencieraient-elles l'une de l'autre? Donc la proposition selon laquelle on a affaire au degré $\mathrm{n}^{\circ} 5$ de rouge ne peut pas être décomposée de cette façon. Et je ne peux donc pas avoir une proposition

10. Wittgenstein, I985, p. 32 (c'est moi qui souligne); trad. fr. p. 26-28.

11. Wittgenstein $1989, \mathbb{} 76$ (c'est moi qui souligne); trad. fr. p. I02. Le tapuscrit des Philosophische Bermerkungen (TS 209) ne présente aucune division en chapitres ou sections. Cette division a été ajoutée par Rush Rhees. J'utilise sa numérotation des remarques pour renvoyer le lecteur aux textes. 
concluante, telle que c'est là tout le rouge qui est disponible dans cette couleur; car cela n'a pas de sens de dire qu'il n'y a plus de rouge à y ajouter, puisque je ne pouvais pas additionner des quantités de rouge par le «et» logique.

Nous avons clairement là une critique de la conception tractatuséenne des nombres. Pour s'en rendre compte, il suffit de se rappeler que tout énoncé employant des nombres devrait être analysé dans le Tractatus comme un énoncé quantifié dans lequel les nombres ne sont pas présents. D’autre part, tout énoncé quantifié devrait être analysé en un produit logique ou une somme logique d'un groupe de propositions choisies selon des critères formels dans lesquels les quantificateurs ne se seraient plus présents.

Revenons par exemple à la manière par laquelle le Tractatus traiterait des propositions comme: "Il y a exactement 2 hommes dans cette chambre. » Wittgenstein l'analyserait précisément comme Frege et Russell, sauf qu'une convention au sujet de l'utilisation des variables remplacerait les énoncés d'identité. En laissant de côté tous les détails qui se rapportent à l'analyse effective des termes tels que "hommes" et "chambre", l'énoncé prendrait la forme suivante:

$$
(\exists x, y) \ldots \& \sim(\exists x, y, z) .
$$

Maintenant, tout énoncé de la forme $(\exists(\exists x) \varphi x$ doit être analysé en une somme logique. La fonction propositionnelle $\phi x$ est associée à une des trois méthodes admissibles de choix énumérées dans 5.50I. Si nous dénotons la totalité de ses valeurs par $[\varphi \bar{x}]$, alors $N[N[\varphi \bar{x}]]$ nous donnera l'affirmation existentielle. Ainsi, une fois qu'on aurait analysé l'énoncé «il y a exactement 2 hommes dans cette chambre", on ne trouverait plus de nombre du tout. Sa construction commencerait par l'ensemble de propositions élémentaires et continuerait étape par étape en n'utilisant rien d'autre que des opérations de vérité (telles que $\mathrm{N}$ ) et des méthodes de choix formellement admissibles (comme celle que nous venons d'employer).

Et qu'en est-il de propositions empiriques générales enveloppant des nombres, telles que: "Il y a plus de personnes dans la chambre B que dans la chambre A »? Faudrait-il quantifier sur des nombres ou alors nier l'existence d'une certaine sorte de bijections? Pas du tout. Il faudrait tout simplement considérer certaines séries formelles d'énoncés, comme:

Il n'y a personne dans la chambre A, et au moins une dans la chambre B.

Il y a exactement une personne dans la chambre A, et au moins deux dans la chambre $\mathrm{B}$.

Il y a exactement deux personnes dans la chambre A, et au moins trois dans la chambre B.

Etc. 
Cette série-là est une série formelle de propositions, puisqu'elle enveloppe une règle récursive. La série a un premier membre et aussi une procédure formelle définie pour obtenir un nouveau membre à un point quelconque. Ainsi, selon 5.50I, je peux associer une variable $\xi$ à ces propositions et prendre la totalité $(\xi)$ de ses membres. L'énoncé $N[N[(\xi)]]$ affirmera donc qu'au moins un membre de la série est vrai, c'est-à-dire qu'il y a plus de personnes dans la chambre B que dans la chambre A.

Ramsey est peut-être le premier à avoir utilisé des séries formelles pour énoncer l'existence d'une infinité d'individus infinis d'une certaine sorte ${ }^{12}$, mais l'énoncé pourrait être certainement construit avec la seule utilisation de la trousse à outils tractatuséenne. Si l'on prend toutes les propositions de la série

$$
\sim(\exists x) \varphi x,(\exists x) \varphi x,(\exists x, y) \cdot \varphi x \cdot \varphi y, \text { etc. }
$$

et si on les nie simultanément, on n'énonce pas une contradiction et l'on dit indirectement qu'il y a un nombre infini d'objets satisfaisant la fonction $\phi x$. Le Tractatus n'a rien contre des infinités actuelles. C'est tout à fait le contraire. Il y a deux méthodes de choix spécifiquement conçues pour embrasser un nombre infini de propositions d'un seul coup, et il y a même une manière de parler de l'infini qui n'oblige pas à considérer l'infini comme un nombre.

Les nombres ne sont employés dans la langue ordinaire et dans le symbolisme logique que pour indiquer combien de fois une certaine opération a été appliquée à un certain point d'une série formelle de propositions. Prenons encore une fois la série formelle:

$$
\sim(\exists x) \varphi x,(\exists x) \varphi x,(\exists x, y) \cdot \varphi x \cdot \varphi y, \text { etc. }
$$

Si nous appelons $\Omega$ l'opération qui produit la série, alors nous pouvons la réécrire de la manière suivante:

$$
\begin{gathered}
\Omega^{0}{ }^{\prime}[\sim(\exists x) \varphi x] \\
\Omega^{1}{ }^{\prime}[\sim(\exists x) \varphi x] \\
\Omega^{2}{ }^{2}[\sim(\exists x) \varphi x] \\
\text { etc. }
\end{gathered}
$$

Les nombres ne sont que des exposants utilisés dans une notation (un symbolisme spécial) susceptible de rendre visible la place qu'une proposition occupe dans une série formelle commençant par une certaine «base» (dans notre cas, " $\sim(\exists x) \varphi x ")$ et définie par une certaine loi récursive de transformation (dans notre cas, $\Omega$ ). Nous pouvons appeler des symbolismes spé-

12. Ibid., $\mathbb{1} 35$; trad. fr., p.I49. 
ciaux de ce genre des «notations $\Omega$ ». Chaque proposition de ce symbolisme pourrait être écrite sans employer des nombres. Au lieu de « $\Omega^{2}$ ' $[\sim(\exists x) \varphi x] »$, par exemple, nous pourrions avoir écrit $(\exists x, y) \varphi x \cdot \varphi y$. Il n'est pas nécessaire d'employer le mot "deux" afin de dire qu'il y a au moins deux personnes dans cette chambre. Les quantificateurs imbriqués peuvent faire exactement le même travail. Mais une fois que j'emploie des nombres, c'està-dire, une fois que j'emploie un symbolisme dans lequel des propositions peuvent être montrées comme le résultat d'applications successives d'opérations récursives sur une certaine base, alors des équations peuvent être employées pour réaliser les déductions logiques qui se fondent sur les propriétés formelles de ces séries. Par exemple, si j'ai I I pommes et je veux donner exactement 3 pommes à chaque personne que je rencontre, combien de personnes obtiendront 3 pommes de moi ? Le calcul avec des nombres me permet de donner la réponse presque immédiatement. Mais le problème est purement logique et n'a rien à voir avec des nombres. Notre problème est celui de remplir la forme

$\mathrm{Si}$ je prends I I pommes et je donne exactement 3 pommes à chaque personne que je rencontre, alors exactement $n$ personnes obtiendront 3 pommes de moi.

avec un nombre $n$ qui transforme la proposition résultante en une tautologie. Et encore une fois la référence aux nombres est tout juste une manière abrégée de faire référence aux structures quantificationnelles. Notre problème logique original serait donc de trouver la structure quantificationnelle correcte à placer à un certain point d'une formule afin de la transformer en une tautologie. Nous plaçant d'emblée au bout de ce raisonnement, nous ne trouverons même pas des quantificateurs, mais seulement des sommes logiques et des produits logiques construits (en dernière analyse) à partir de propositions élémentaires. Comme nous l'avons déjà dit, dans le Tractatus il n'y a aucun nombre «à la base du langage ". Les nombres sont tout juste un soutien à la faiblesse humaine apparaissant dans beaucoup de langages naturels et également dans des symbolismes spéciaux comme la notation $\Omega$ présentée par le Tractatus.

Frege et Russell pourraient facilement convenir qu'après tout les nombres ne sont que des "soutiens à la faiblesse humaine». Mais si nous regardons d'un peu plus près, les différences prennent le devant. Bien qu'ils conviennent tous les deux qu'idéalement nous pourrions parler sans employer des nombres, ils incluraient dans la liste de propositions de notre langage des équations comme " $5+7=\mathrm{I} 2$ » qui selon Wittgenstein ne sont pas des "propositions significatives» - du moins pas en tant qu'équations ou propositions arithmétiques. Qu'en est-il de $" 5+7=\mathrm{I} 2$ » dans le Tractatus? Il s'agit tout simplement d'une règle de substitution. Elle dit (ou au moins essaye de dire) que le signe " I 2 " peut être substitué au signe " $5+7$ " dans n'importe quel énoncé sans changement de la signification. Sans cette dernière clause, elle serait complètement analogue à une règle des échecs, et il 
n'y aurait en elle rien de plus mystérieux ou d'indicible que dans ce qui doit être contenu dans n'importe quel énoncé contenant le mot "peut». On pourrait peut-être la réduire, par exemple, à la description anthropologique d'une coutume. Nous avons l'habitude de déplacer les pièces d'échecs (ou de nous servir de la fourchette et du couteau) de telle et telle manière, et également l'habitude de dessiner les signes mathématiques selon tels et tels modèles. Ne pouvons-nous pas décrire nos propres coutumes? Apparemment oui. Tout le problème se trouve donc dans cette clause finale — «sans changement de la signification ». Seule cette clause peut donner à cette règle le statut d'une règle mathématique (et, selon le Tractatus, purement logique). Mais c'est justement cette clause finale qui est la partie "ineffable» de la règle — l'élément qui ne pourrait jamais être réduit à la simple description anthropologique d'une coutume. L'identité de la signification n'est pas quelque chose que je peux décrire. Elle est quelque chose qui doit se montrer dans le seul langage qu'il me soit possible de comprendre: le langage dans lequel je pense.

La logique nous montre pourquoi «I 2 " peut être substitué à " $5+7$ " dans n'importe quel énoncé de notre langage sans changement de signification. Dans un certain sens, elle nous donne une raison ineffable d'accepter comme logiquement nécessaire un résultat ineffable. Nous n'avons qu'à nous rendre compte que des nombres sont employés dans notre langage ordinaire (et également dans la notation $\Omega$ ) pour placer des signes propositionnels dans le contexte d'une série infinie de signes propositionnels. La notation $\Omega$ rend cela plus visible. Ainsi, la proposition «Il y a I 2 personnes dans cette chambre» peut être représentée sous la forme $O^{12}{ }^{\prime} p$, si nous prenons $p$ comme la proposition qui dit qu'il n'y a personne dans cette chambre et $O$ comme la transformation formelle qui produit des séries comme - «il n'y a aucun...», «il y a exactement un...», etc. Mais nous pourrions prendre $\mathrm{O}^{7}$ ' $\mathrm{p}$ comme le premier membre d'une nouvelle série. Si nous employons la même opération $O$ pour produire de nouveaux membres en partant de $O^{7} p$, alors il est logiquement nécessaire que $O^{5} \mathrm{O}^{7} \mathrm{p}$ soit la même proposition que $O^{12}$ 'p (en fait, qu'elle soit exactement le même signe propositionnel). Dans ces notations, le signe «I 2 » peut être substitué au signe " $5+7$ " comme exposant d'une opération. Tel est l'emploi que nous faisons d'une équation comme " $5+7=\mathrm{I} 2$ ".

Wittgenstein soutient dans l'article sur la forme logique (et également en $P B V I I I)$ que les quantificateurs ne peuvent pas être employés pour exprimer la mesure. Si nous essayons de le faire, nous sommes condamnés ou bien à détruire l'étalon (qui doit être unique et non pas multiple), ou bien à dissoudre toutes les mesures dans l'unité. Ainsi, Wittgenstein nie décidément qu'il soit possible de mettre une série de propositions exprimant des mesures comme

Cette table a un I mètre de long.

Cette table a 2 mètres de long. 
Cette table a 3 mètres de long. et ainsi de suite,

sous la forme

$$
\begin{aligned}
& \Omega^{0}{ }^{\prime}(E ! 1 x) \varphi x \\
& \Omega^{0}{ }^{\prime}(E ! 2 x) \varphi x \\
& \Omega^{0}{ }^{\prime}(E ! 3 x) \varphi x
\end{aligned}
$$

et ainsi de suite.

Si ces propositions sont disposées dans une série formelle (comme elles semblent l'être), aucune d'elles ne pourrait être analysée en une proposition dans laquelle les quantificateurs remplacent les nombres en exerçant les fonctions de ceux-ci. Des nombres doivent être présents à la fin même de l'analyse, et non seulement dans des énoncés physiques (hypothétiques) au sujet d'objets physiques, comme les tables. On doit aussi s'attendre à leur présence dans n'importe quelle attribution de couleur à une tache dans mon champ visuel. Les nombres ne sont pas simplement un expédient notationnel pour marquer l'emplacement d'une proposition dans une série qui se développe selon une règle récursive. Tout d'abord, ils sont des composants des propositions elles-mêmes, tout à fait indépendamment de leur insertion dans telle ou telle série. Ils font partie des outils de base du langage, et les règles de substitution comme $" 5+7=\mathrm{I} 2$ » ne sont pas des idiosyncrasies de certaines notations, mais doivent être disponibles au niveau élémentaire si les propositions doivent avoir la multiplicité nécessaire pour exprimer des événements phénoménaux comme la simple présence d'un cercle rouge dans mon champ visuel. Si je vois un cercle rouge avec 5 degrés de luminosité et que ce cercle devient plus lumineux de 7 degrés, alors I 2 degrés s'ensuivront comme conséquence logique directe. Si je demande combien de degrés de luminosité aura cette tache rouge après être devenue 7 degrés plus lumineuse, je ne suis pas (et ne pourrait pas être) en train de me demander quelle est la structure quantificationnelle correcte à employer. Je m'enquiers d'un nombre et de rien d'autre. Ce que Wittgenstein annonce dans les Bemerkungen n'est rien de moins que l'abandon de l'aspect réductionniste du logicisme que le Tractatus a cherché après tout à conserver. C'est à mon avis l'axe autour duquel la philosophie de la période intermédiaire peut être rationnellement reconstruite.

Prenez par exemple la "phénoménologie» qui occupe les huit premiers et les trois derniers chapitres des Bemerkungen. Tout ce qui y est dit au sujet de "phénomènes" est-il une vraie nouveauté, ou Wittgenstein explicite-t-il tout simplement quelque chose de constitutif pour sa première philosophie, qu'il est incliné à préserver dans la période intermédiaire? Je pense que cette question est trompeuse, car l'enjeu fondamental tend par là à être caché par la tentation de «deviner» les intentions de Wittgenstein, ou de trouver «la seule solution possible». La vraie question est: "Pourquoi 
Wittgenstein pense-t-il qu'il peut passer sous silence la nature des propositions élémentaires dans le Tractatus, alors que dans la période intermédiaire l'analyse effective remplace les seuls principes généraux? » Tel est le problème réel. Même s'il était vrai que Wittgenstein «n'aurait dans le Tractatus aucune autre solution" que d'admettre les phénomènes comme matériau de base de toute description possible, il n'aurait néanmoins par là aucune raison spéciale de devenir un "phénoménologue». Exhumer la totalité des propositions élémentaires pourrait être une tâche importante en elle-même, mais cela n'ajouterait jamais rien de nouveau à la provision de relations logiques entre des propositions. Aucune «nouvelle tautologie» (ni «nouvelle contradiction ") ne serait dégagée par là. Des vérités logiques pouvaient être établies à l'avance parce qu'elles étaient basées sur les seuls connecteurs vérifonctionnels. C'est pourquoi nous pouvions écrire nos tables de vérité habituelles en utilisant les lettres schématiques $p$ et $q$, sans mentionner le genre de proposition que ces lettres sont censées remplacer. Quoi qu'il en soit, peu importe. C'est à cela que revient l'indépendance mutuelle des propositions élémentaires.

Dorénavant, les propositions élémentaires ne sont plus inconditionnellement indépendantes les unes par rapport aux autres. On cite d'habitude l'exclusion des couleurs comme le meilleur exemple de ce changement. Une tache dans le champ visuel ne peut pas être rouge et verte en même temps, et elle ne peut être rouge et jaune en même temps que dans le sens très spécial d'être une tache rouge jaunâtre. Ainsi, une proposition qui dit que si une tache est rouge alors elle n'est pas verte doit être tout autant une tautologie qu'une proposition qui dit que si elle est rouge alors elle est rouge. Celle-ci ne dépend que du fonctionnement d'un connecteur, mais celle-là dépend de la structure logique des couleurs. Toutefois, ici nous devons être prudents. La structure logique des couleurs est tout juste un cas particulier de ces "propriétés qui admettent une gradation » et qui doivent être représentées par des nombres associés à des systèmes de coordonnées. Rappelons-nous le texte que nous avons cité un peu plus haut:

[L]es nombres doivent intervenir dans ces formes lorsque - ainsi que nous le dirions dans la langue ordinaire - nous traitons de propriétés qui admettent une gradation, par exemple des propriétés comme la longueur d'un intervalle, la hauteur d'un son, la luminosité ou la rougeur d'une nuance de couleur, etc.

De même que la théorie physique représente l'exclusion mutuelle de couleurs au moyen d'attributions incompatibles de nombres, ainsi doit-il en être pour ce qui est des rapports phénoménaux. Si nous assignons un «nombre-de-couleur» (ou un triplet de nombres) à un certain endroit de notre champ visuel, nous ne pouvons pas assigner un nombre différent (ou un triplet) au même endroit. Si je dis qu'il y a exactement 5 personnes dans cette chambre, je peux immédiatement en déduire que le nombre de personnes dans cette chambre n'est pas 7. Si cette pièce a 5 mètres de long, elle 
ne peut pas avoir 7 mètres de long. Si la couleur d'une tache est associée au numéro 5 , cette même tache ne peut pas en même temps avoir la couleur associée au numéro 7. L'exclusion mutuelle de couleurs est tout juste un cas d'exclusion mutuelle des attributions numériques. C'était la doctrine «sousentendue ", sinon explicitement indiquée dans le Tractatus. La différence, comme nous l'avons vu, est que la grammaire des nombres était fournie dans le Tractatus par le comportement logique des quantificateurs imbriqués: 5 est incompatible avec 7 parce que $(E ! 5 x) \varphi x$ est incompatible (vérifonctionnellement incompatible!) avec' $E ! 7 x) \varphi x$. Après I929, Wittgenstein se rend compte que les attributions de nombre doivent être considérées comme immédiatement incompatibles les unes avec les autres. Les quantificateurs n'ont rien à nous dire au sujet des raisons logiques impliquées ici. Les nombres doivent prendre soin d'eux-mêmes.

Dès lors, on comprend pourquoi la phénoménologie a une telle importance dans les Bemerkungen. Même si une proposition n'est pas construite à partir de propositions plus simples au moyen de connecteurs logiques, elle a une logique qui lui est propre. Nous ne pouvons pas déterminer le domaine des inférences logiques en ne prêtant attention qu'aux seules propositions moléculaires. Il y a tout un réseau de relations logiques qui s'établissent entre les propositions élémentaires, et ainsi la logique ne peut pas éviter les peines de l'analyse logique. Sans savoir quelles propositions élémentaires il $\mathrm{y}$ a, nous ne saurons jamais à quoi les tautologies ressemblent, c.-à-d., quelles formes logiques elles peuvent assumer. C'est pourquoi une bonne partie des Bemerkungen est consacrée à l'analyse logique des phénomènes. Il ne s'agit pas simplement d'employer les outils logiques dans une tâche vaguement philosophique. Il s'agit bien plutôt d'employer l'analyse logique comme la seule manière d'obtenir certains résultats strictement logiques.

Les nombres ne sont pas appliqués uniformément partout. Quand ils sont employés pour mesurer, ils peuvent être associés à un seul système de coordonnées ou bien à deux, trois, etc., systèmes de coordonnées. Comme nous l'avons vu, un énoncé très simple comme «il y a un cercle rouge dans mon champ visuel » peut être vu comme la synthèse de trois mesures: deux pour le cercle et une troisième pour la couleur. Un cercle dans mon champ visuel peut être déterminé par une paire de coordonnées pour la position de son centre et par un nombre donnant la mesure de son rayon. Dans un système assez grossier de représentation, nous pourrions également associer chaque couleur du spectre à un nombre rationnel avec une certaine marge de variation. C'est là exactement le genre de représentation qui est donnée de façon figurative au chapitre VIII des Bemerkungen. À ce moment-là, Wittgenstein essaye de prouver que

C'est mal re-présenter la chose que dire d'un objet qu'on n'a pas le droit de lui conférer deux attributs qui seraient inconciliables. En effet, il semble alors que l'on doive en chaque cas rechercher d'abord si deux déterminations sont ou 
non conciliables. La vérité est que deux déterminations de la même espèce (coordonnées) sont impossibles ${ }^{13}$.

Peu après, Wittgenstein insère le dessin suivant dans le manuscrit

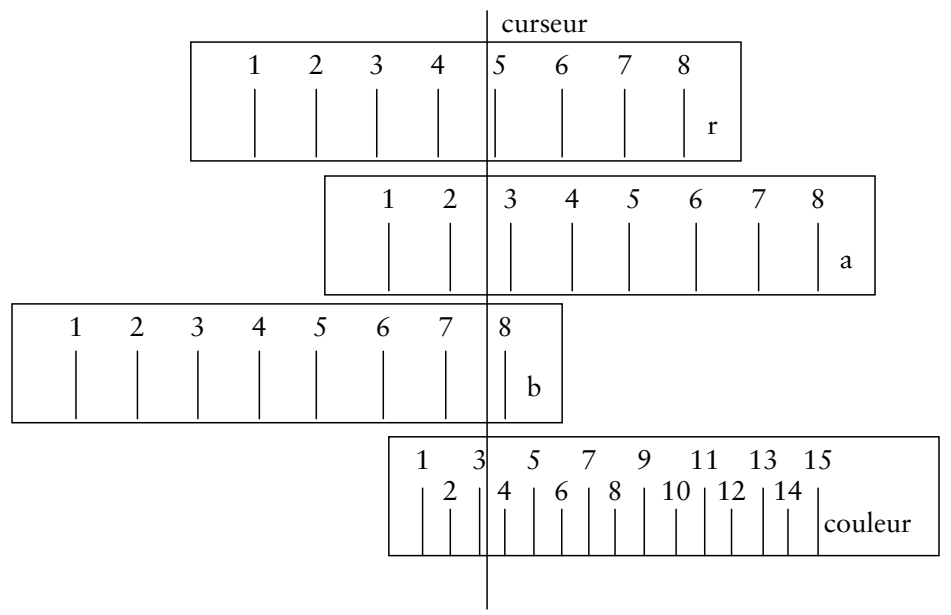

À côté du dessin, il ajoute ce commentaire ${ }^{14}$ : "Cela donnerait par exemple l'indication qu'un cercle coloré de couleur... et de rayon... se trouve en un lieu...»

Je pense que ce qu'il faut noter avant tout dans ce passage, c'est qu'une proposition entière est présentée comme une synthèse des nombres. Si on la couchait par écrit, elle pourrait prendre l'aspect suivant: « $(\mathrm{r},(\mathrm{a}, \mathrm{b})) \mathrm{F}$ », où « $\mathrm{r} »$ serait un nombre donnant la mesure du rayon, « $(\mathrm{a}, \mathrm{b}) »$ serait un couple de nombres donnant les coordonnées du point central du cercle (et ainsi sa position dans le champ visuel), et $\mathrm{F}$ serait un nombre (ou un triplet de nombres) associé à une couleur du spectre. Aucun de ces nombres ne signifierait quoi que ce soit en lui-même. Ils ne pourraient exercer leurs fonctions qu'associés à l'avance à certains systèmes de mesure: l'un d'entre eux devrait pouvoir donner la grandeur d'une ligne indépendamment de sa position dans le champ visuel; un autre devrait déterminer un point spécifique dans le champ visuel au moyen de deux coordonnées; finalement, un troisième devrait associer un nombre défini à chaque couleur du spectre. Dans le contexte de ces systèmes donnés à l'avance, la synthèse de toutes ces mesures serait exactement une proposition comme «il y a un cercle rouge de telle et telle grandeur dans telle et telle position de mon champ visuel».

$$
* * *
$$

13. Ibid., $\mathbb{S} 84$ (c'est moi qui souligne); trad. fr. p. Io8.

14. On trouve ce dessin dans les manuscrits, et Wittgenstein a écrit «Farbe» («couleurs») sur la dernière règle. Voir Wittgenstein's Nachlass (Bergen Electronic Edition), MS ıо8, p. 54. 
Toute cette évidence textuelle puisée au chapitre VIII doit être réconciliée d'une façon ou d'une autre avec la déclaration explicite faite au chapitre XX: «Dans l'espace visuel il n'y a pas mesure ${ }^{15}$.»

Le travail éditorial de Rush Rhees sur le TS 209 a ajouté des divisions en chapitres et sections à un texte originellement continu ${ }^{16}$. Ce fut vraiment du bon travail - un ajout qui est en même temps anodin pour le spécialiste et utile à l'étudiant. Grâce à ce travail, nous pouvons facilement voir une particularité structurale troublante des Philosophische Bemerkungen. Après s'être appesanti sur les fondements des mathématiques du chapitre X au chapitre XIX, Wittgenstein revient brusquement au sujet du chapitre VIII, à savoir - les couleurs et la structure de l'espace visuel. Tel est le thème des chapitres XX et $\mathrm{XXI}^{17}$. Quels sont la signification et le but de cette reprise?

Nous pourrions essayer de faire appel à un motif fortuit - comme on le sait, Wittgenstein a rassemblé le texte en toute hâte afin de soulever les fonds "nécessaires à la poursuite de son travail ${ }^{18}$ ". Pourquoi ne pas blâmer les dates limites fixées par le conseil ? Pourquoi ne pas imaginer que, après avoir dicté à un dactylographe un choix de remarques puisées dans les MS I05-I08, Wittgenstein les ait organisées selon leur sujet, mais ait laissé de côté quelques remarques importantes au sujet de la grammaire des couleurs et de la structure de l'espace visuel. Ceux-ci ont été alors placés à la fin d'un livre que - il le savait très bien — pas même Russell ne lirait d'un bout à l'autre.

Tout à fait indépendamment du fait que cela pourrait à peine s'appeler une "hypothèse de lecture ", ce ne semble pas être vrai même pour ce qui serait un fragment biographique. Une comparaison superficielle des Bemerkungen avec les manuscrits qui lui ont donné origine suffit pour montrer le soin obsessif avec lequel Wittgenstein a rassemblé le texte. Une des vertus des divisions introduites par Rhees est de rendre explicite la structure soigneusement élaborée du texte. Des thèmes tout à fait reconnaissables sont alignés dans un ordre parfaitement justifiable. Le texte est loin d'être facile, mais il est encore plus loin d'être brouillon. D'ailleurs, si Wittgenstein voulait placer les chapitres finals au début du livre, cela aurait été aussi facile que de mettre un document dans une chemise. Nous devons être plus charitables avec notre philosophe. Il ne pouvait pas être si paresseux.

Ainsi, les chapitres finals des Bemerkungen présentent ce que nous pourrions appeler un «problème d'emplacement»: pourquoi ont-ils été placés exactement là? Mais il y en a un autre, plus difficile à résoudre. Si nous comparons le chapitre XX et le chapitre VIII, par exemple, ils semblent

15. Wittgenstein $1989, \mathbb{2 1} 2$; trad. fr. p. 253.

16. Voir la «Remarque de l'éditeur » à la fin du livre (pour la trad. fr., au début du livre).

17. Le chapitre XXII s'occupe de la notion d' «hypothèse ", et de sa fonction en science. Cela aussi est une reprise d'un sujet déjà abordé par Wittgenstein au début du livre.

18. Monk, I99I, p. 29I. 
se contredire. Dans le chapitre VIII, Wittgenstein a insisté sur le fait que nous devrions voir la proposition comme un étalon de mesure appliqué à la réalité. Plus précisément, la proposition devrait être regardée comme une «synthèse» de mesures. Au $\mathbb{\$} 84$, il présente même une représentation graphique de cette idée, que nous avons reproduite plus haut: quatre de ces règles sont employées pour exprimer la proposition «il y a un cercle rouge à tel et tel point de mon champ visuel». Trois de ces règles définiraient numériquement la forme, la taille et la position du cercle, et l'autre présenterait numériquement une certaine couleur. Wittgenstein semble proposer que les descriptions de l'espace visuel puissent (en principe) être données dans un "langage de coordonnées" où des nombres sont employés pour caractériser des formes (comme le cercle), les situant à l'intérieur du champ visuel, et aussi pour caractériser la couleur que nous attribuons à cette forme.

Les chapitres finals du livre semblent aller exactement dans la direction opposée. Là, Wittgenstein essaye de prouver que nous ne pourrions pas appliquer un système de coordonnées au champ visuel et que l'idée de mesure est logiquement incompatible avec celui-ci. Voici un passage typique à cet égard ${ }^{19}$ :

Le mot "égalité » a une autre signification, appliqué à des segments de droite dans l'espace de notre vision [Sehraum], que celle qu'il a lorsqu'il est appliqué à des segments du monde physique. L'égalité, dans l'espace de la vision, a une autre multiplicité que l'égalité dans l'espace physique; c'est pourquoi dans l'espace de la vision gI et $g_{2}$ peuvent être des droites (des droites vues [Sehgerade]) et on peut avoir les traits $\mathrm{a}_{\mathrm{I}}=\mathrm{a} 2, \mathrm{a}_{2}=\mathrm{a}_{3}$, etc., mais non $\mathrm{a} \mathrm{I}=\mathrm{a} 5$.

De telles situations sont analogues à celles en cause dans la question: combien de grains de sable sont nécessaires pour faire un $\operatorname{tas}^{20}$ ? Quand nous décrivons deux distances phénoménales en tant que phénoménalement identiques, nous sommes obligés d'employer le mot «identique » selon des règles incompatibles avec le sens dans lequel ce mot doit être employé dans le contexte de la mesure. Il est impossible de parler de la «mesure» comme nous le faisons habituellement si notre concept d' «identité » n'est pas inconditionnellement et universellement transitif. Confrontés à une situation semblable dans le monde physique, nous serions amenés à faire des mesures plus précises, à employer des instruments plus précis, à être plus patients, plus soigneux, et ainsi de suite. Chacun de ces procédés est exclu du monde des phénomènes. Là, "regarder de plus près " n'a aucun sens. Les choses sont exactement ce qu'elles semblent être. S'il me semble que les distances sont telles que $\mathrm{a}_{\mathrm{I}}=\mathrm{a}_{2}, \mathrm{a}_{2}=\mathrm{a}_{3}$, etc., mais non $\mathrm{a}_{\mathrm{I}}=\mathrm{a}_{5}$, alors les choses sont ainsi, et c'est tout. Le signe d'égalité utilisé dans les mesures physiques n'a aucun

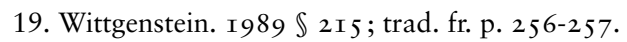

20. Ibid., $\mathbb{} 2 \mathrm{II}$; trad. fr. p. 250 . 
emploi dans le champ visuel. Nous ne pouvons pas "mesurer» l'espace de la vue comme nous mesurons la distance entre deux objets physiques.

Il est difficile d'évaluer ce contraste entre la fin et le début des Philosophische Bemerkungen en ce qui concerne la mesure et les nombres. Je ne prétends pas avoir de solution à ce problème. Mais j'aimerais suggérer que c'était là un problème pour Wittgenstein lui-même, et qu'il faisait intégralement partie de l'ordre du jour philosophique inscrit dans le livre. Je ne peux voir aucune autre raison au fait qu'il place à la fin de son texte des observations comme celle que nous venons tout juste de citer. Je les prends comme l'aveu de Wittgenstein qu'il faisait face à un problème non résolu - un problème qui appelle de futures recherches, à être menées après la conclusion du livre.

Une chose est certaine. Il n'y a aucune voie de retour vers le Tractatus. Les nombres ne sont pas réductibles aux quantificateurs imbriqués - cela est définitif, et tout le traitement de l'arithmétique présenté dans le livre en témoigne. L'arithmétique est grammaire ${ }^{21}$, et elle est "suffisamment fondée sur elle-même ${ }^{22}$ ». Elle n'a pas besoin de (et ne pourrait absolument pas) trouver son fondement ailleurs - dans la logique des fonctions de vérité, par exemple. L'arithmétique doit prendre soin d'elle-même, comme tout ce qui appartient au domaine de la logique. La relation entre les nombres et les phénomènes est sûrement problématique, et Wittgenstein devra affronter ce problème. Comme on le sait, les phénomènes seront bientôt mis hors de considération, tandis que l'idée que l' "arithmétique est (irréductiblement) grammaire » demeurera. Le chemin par lequel Wittgenstein est arrivé à cette position est encore à découvrir.

Traduit de l'anglais par Bento Prado Neto

\section{Bibliographie}

Copi, Irving M., and Robert W. Beard (eds). Essays on Wittgenstein's Tractatus, London, Routledge and Kegan Paul, 1966.

Frascolla, P. Wittgenstein's Philosophy of Mathematics, London, Routledge, I994, chapitre I.

Marion, M. Wittgenstein, Finitism and the Philosophy of Mathematics, Oxford, Clarendon Press, I998.

Monk, R. Ludwig Wittgenstein: The Duty of Genius, London, Vintage, I991.

Potter, M. Reason's Nearest Kin, Oxford, University Press, 2000.

Wittgenstein, L. "Some Remarks on Logical Form », in Ludwig Wittgenstein. Philosophical Occasions, 1912-1951, James C. Klagge and Alfred Nordmann (eds.), Indianapolis, Hackett Publishing, I993; trad. fr., Quelques remarques sur la forme logique, T. E. R, 1985.

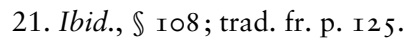

22. Ibid., $\mathbb{S}$ Io9; trad. fr. p. I25. 


\section{Philosophiques / Printemps 2012}

Wittgenstein, L. Philosophische Bemerkungen, Werkausgabe Band 2, Suhrkamp, I989; trad. fr., Remarques philosophiques, Gallimard, I975.

Wittgenstein, L. Tractatus Logico-Philosophicus, translated by B. F. McGuiness, and D. Pears, Routledge, 2004; trad. fr. Tractatus logico-philosophicus, Gallimard, I993.

Wittgenstein, L. Wittgenstein's Nachlass, The Bergen Electronic Edition, Oxford, 2000. 